\title{
The application of dried blood spot sampling in global clinical trials
}

“...clinical trials may see a substantial savings in costs and an increase in overall quality when a number of pharmacokinetic or biomarker samples can be collected, and thus analyzed, on dried blood spot cards, rather than as a plasma sample."

\begin{abstract}
Keywords: blood $\approx$ clinical trial $\approx$ costs $\approx$ DBS $\approx$ dried blood spot $\approx$ dry ice $\approx$ logistics $\approx$ plasma $\approx$ quality - safety $=$ sampling = shipment
\end{abstract}

Clinical trials in patients often require a large amount of sampling from the participating volunteers. Prior to inclusion in the study, blood samples are taken to be able to judge whether or not the volunteering patient's condition complies with the criteria in the protocol and often samples are also drawn to achieve baseline values of a number of parameters. During the trial, patients visit their physician for further examination and it is not uncommon to draw a number of blood samples during those visits. Depending on the phase of the program and the type of drug under development, the number of blood samples collected during a clinical study can range from a few hundred to many thousands. As a rule, all these samples need to be shipped to specialized laboratories to be analyzed. The amount of work involved around sample collection, shipment and analysis is quite substantial and a significant time and cost factor in regulated drug development.

The concept of dried blood spot (DBS) sampling, collecting a capillary blood sample from pricking the heel or a finger, was introduced in Scotland in 1963 by Robert Guthrie to enable screening for metabolic diseases in large populations. Neonatal screening was implemented nationwide from the late 1960s onwards in many countries in the western world. While it originally only concerned testing for phenylketonuria, the number of tests has been expanded to hypothyroidism, sickle cell disorders and HIV. Alongside neonatal screening there are an increasing number of tests made available for ambulant patients. This concerns measurements of, for example, a number of hormone levels as well as therapeutic drug monitoring [1].

In contrast, there never seemed to be much interest in applying DBS in drug development, but this may quickly change since DBS was on the agenda of a number of recent meetings [2-4].
So far, it mostly concerns animal pharmacokinetic and toxicokinetic studies; the advantages of DBS here are in the refinement and reduction of animal experiments. DBS in patient trials has only or mainly been in the area of antimalaria drugs, inferred from the above-mentioned meeting. Application of DBS for pharmacokinetic (PK) objectives in human volunteer studies has recently been reported by Spooner et al. [5] .

The less frequent application in clinical trials is not so surprising given the relatively short period that DBS has been under the attention of drug developing companies. Furthermore, testing the value of a new approach would logically start in the area where studies are of relative short duration and relatively low cost, thus minimizing the risk of a less favorable outcome. And, quite importantly, the scientific community in drug development are very much accustomed to working with plasma or serum, so have to analyze and understand the impact that a switch to blood may have on their programs [6]. Trials utilizing DBS accounted for less than $2 \%$ of the trials in 2009 for Quintiles Laboratories, GA, USA [Unpublished Data].

Given the limited practical experience with DBS in large-scale patient trials, analyzing advantages of DBS versus traditional sampling is unfortunately still largely a theoretical exercise. By comparing the general steps and aspects of a multinational multisite clinical study for DBS to traditional tube-based sampling, a good overview of the potential advantages and challenges can be achieved.

First let us define an 'average' late-stage clinical study. This study will include 50 investigator sites, spread over ten countries and will in total recruit 500 patients. Per patient there will be five visits in which two safety samples, five biomarker samples and five PK samples are collected. Safety

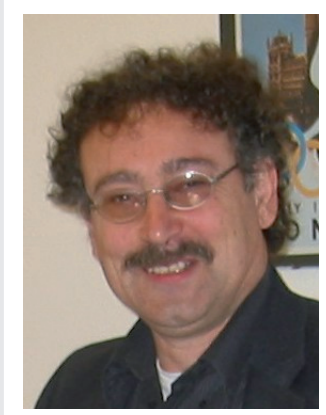

Peter van Amsterdam

Abbott Healthcare Products BV, Postbox 900, I380 DA Weesp C.J. van Houtenlaan 36, Netherlands

Author for correspondence: Tel.: +3l $29447769 \mid$

Fax: +31 294477 |4|

E-mail: peter.vanamsterdam@ abbott.com

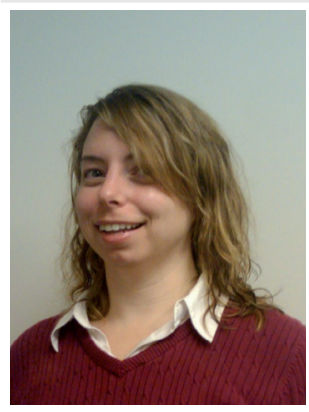

Clare Waldrop

Quintiles Laboratories Ltd, Marietta, GA 30067, USA 
samples need to be shipped on the day of collection to a central laboratory. Some biomarker and PK samples may also need frequent and rapid shipments while others can be saved until the end of the study. Whereas safety samples can be shipped at ambient temperature, biomarker and PK samples often require storage in deep freezers and shipment on dry ice. Allowing for some gain in efficiency by combining shipments and temporary storage of a number of biomarker or PK samples at the site, we assume a 2000 shipments of safety samples at ambient conditions and 1000 on dry ice in our example study.

\section{"...there never seemed to be much interest in applying dried blood spots in drug development, but this may quickly change as dried blood spots has been on the agenda of a number of recent meetings."}

The following steps and aspects are relevant: training of clinical study site staff on sampling and sample shipping, kit supply and shipping, sample processing, interim sample storage at investigator site, sample shipments to central facility, storage at central and bioanalysis laboratory, sample handling and sample destruction.

\section{Study staff training}

Pharmacokinetic and biomarker samples usually require millilitres of whole blood be drawn in a dedicated tube, centrifugation under specified conditions and cautiously transferring the upper plasma layer or pipetting a predefined volume into another tube. In addition, it may be necessary to add stabilizing agent(s), for instance an antioxidant. Study staff need to be trained to ensure correct execution of those steps.

In a DBS scenario, the blood draw - a simple finger prick - becomes much simpler and the centrifugation step and transferring the plasma layer are not applicable anymore. Furthermore, it has been reported by many speakers that analytes on the DBS card appear to be more stable than in the comparable frozen matrix. A shorter and simpler training, less complicated sampling and better adherence to the study plan are clear advantages reducing the costs and increasing the quality. We estimate the direct cost savings to be small, but the potential quality increase is quite a significant factor. We have seen sites that took PK draws from an indwelling needle used to administer the drug. With a finger prick this level of contamination is not possible.

\section{Sample kit building \& shipping}

Sampling kits for standard blood sample require more materials, especially tubes, then for DBS sampling, but as it is now DBS cards are more expensive than standard tube types. When optimized, DBS kits may be smaller in size then tube-based sampling kits, but even by reducing the size and weight of kit sets we do not expect a major shift in costs.

\section{Sample processing}

Acquiring plasma samples requires centrifugation of the blood sample at the clinical site. There are occasions in which there is not even a regular laboratory centrifuge present at the investigator site, when a study requires cooled centrifuges they almost always need to be bought or leased especially for the study. Those costs can run up to hundreds of thousands of euros.

\section{Sample shipping}

Transport of PK and biomarker samples is usually done on dry ice. Dry ice shipments fall under the International Air Transport Association (IATA) class 9 dangerous goods regulation [101], and thus require sites to be trained on IATA compliance and dry ice handling. Dry ice shipments can also be expensive and in some countries it may even be impossible for a study center to acquire dry ice. DBS cards are usually shipped in a sealed bag with a desiccant and given the antimicrobial properties of the DBS sample, do not require special biohazard arrangements [7]. Both aspects - no dry ice and an intrinsic safe sample - imply easier handling, less paper work, smaller and lighter weight packages. Depending on the weight, distance, shipper and local conditions, the costs savings of DBS shipments compared with plasma can be $€ 200$ per shipment. In our example study, depending on the total number of dry ice shipments, total costs savings may add up to $€ 200,000$. Furthermore, we would like to mention that the current experience is that approximately $30 \%$ of the dry ice shipments have issues such as incorrect packaging or incorrectly completed shipping documentation. This causes frustration for the sites and potentially places valuable samples at risk.

\section{Sample storage}

Samples typically need to be stored for a number of days up to a few years before they are analyzed. Biological samples are usually stored at approximately -20 or $-80^{\circ} \mathrm{C}$ and can be required 
at the clinical site, the central laboratory, a dedicated sample repository and/or the bioanalytical laboratory. DBS cards do not necessarily require less storage space, but they do allow storage at ambient temperatures, thus saving electricity and the purchase of freezers.

“...the current experience is that approximately $30 \%$ of the dry ice shipments have issues such as incorrect packaging or incorrectly completed shipping documentation. This causes frustration for the sites and potentially places valuable samples at risk."

An important aspect is that clinics often do not have $-80^{\circ} \mathrm{C}$ freezers, thus, in cases where plasma samples require storage at $-80^{\circ} \mathrm{C}$, samples either need to be shipped on dry ice on a visitby-visit basis or the freezers need to be bought or leased for each of the sites. In both scenarios the additional costs can be hundreds of thousands of euros.

\section{Sample receipt}

Whereas deep frozen shipped samples need to be unpacked and checked upon receipt, processing of received DBS cards can basically be done at any kind of laboratory bench. The advantages for the technicians are clear. We also recognize a saving in the amount of dry ice needed at a laboratory and possibly less demand for costly laboratory space.

\section{Sample analysis}

Processing plasma samples involves a safety risk for the laboratory technicians and, thus, this is usually done in a fume cupboard or a laminar flow cabinet. Working with DBS cards would remove these safety requirements and the advantages, in addition to offering a reduced health risk for laboratory personnel, obviously include savings in laboratory space, flow cabinets and electricity. For a laboratory that can completely change from liquid biological samples to DBS samples the costs saving will be quite substantial.

\section{Sample destruction}

Sample remainders (partly filled tubes) are usually destroyed after completion of the study. Again, here we can see that the proper waste disposal requirements for the DBS cards may be simpler and, thus, cheaper than for plasma samples.

\section{Bioanalytical considerations}

In the previous sections we discussed the aspects relevant with respect to samples. But clearly there are a number of factors that need to be taken into account with respect to method development and validation. Perhaps the most important aspect is that the very low absolute amount of sample that can be processed brings extra challenges regarding sensitivity. Other challenges are the extraction from the spot and on-card stability of labile compounds. Furthermore, as DBS cards are shipped and stored under ambient conditions, stability experiments should take into account a range of possible temperatures. In addition, there is the aspect of automation, which at the moment is not at the level of handling liquid samples. All these aspects imply that the efforts and costs of method development, validation and possibly also routine sample analysis will be higher then they are for plasma samples. Nevertheless, we expect such an impact to decrease with time while the laboratories get more experienced in handling DBS samples.

\section{Future perspective}

The interest for DBS in regulated drug development has grown enormously in only a few years; and the outlook is promising: basically DBS samples can be analyzed as well as plasma or full blood samples, there are ethical benefits with respect to the ' 3 Rs' guiding principles for research involving animals, and substantial cost savings in clinical trials. As with LC-MS/ MS, which was the technical revolution for bioanalysis, DBS has everything in it to be the sampling revolution.

\section{Conclusion}

Our conclusion is that clinical trials may see substantial savings in costs and an increase in overall quality when a number of PK or biomarker samples can be collected and thus analyzed on DBS cards rather than as a plasma sample. For a complicated trial requiring multiple and frequent dry ice shipments and/or cooled centrifuges and/or $-80^{\circ} \mathrm{C}$ freezers on site, the costs savings can easily add up to multiples of $€ 100,000$. A second aspect is that we expect improvements in quality in most of the processes related to sampling. An increased quality is in the benefit of all: sponsor, investigator and especially for the patient. Finally, we see improvements in safety. Not only is a DBS card a far less hazardous sample than plasma or full blood, there is also the benefit of no longer needing to manipulate the sample with dry ice. 


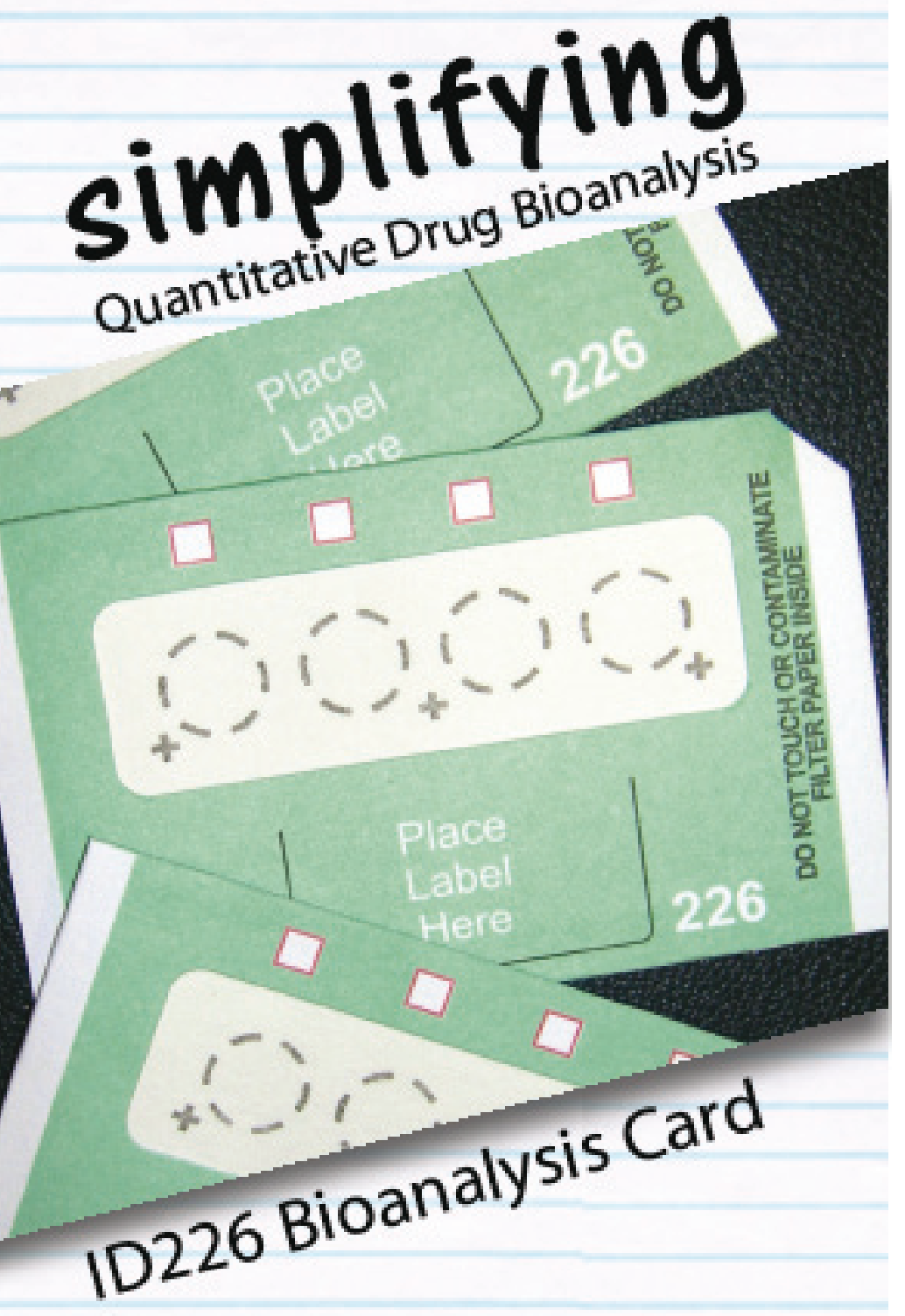

Advantages of ID226 Bioanalysis Cards

$\checkmark$ Cost reductions

$\checkmark$ Clinical advantages

$\checkmark$ Simple method development

$\checkmark$ Superb uniformity

$\checkmark$ Appication versatility

$\checkmark$ Affordable choice

The ID226 Bioanalysis Card has received rave reviews within the pharmaceutical industry and is rapidly becoming the DBS product of choice for quantitative bioanalytical (DMPK) assays.

\section{ID Biological}

www.id-biological.com
17 P and N Drime

Greenville, SC 29611, USA

Tel: (001) 864.299.8787

Fax: $(001) 864.299 .8797$

\section{Disclaimer}

The views expressed in this article are those of the authors and do not necessarily reflect the respective company's position on the subject.

\section{Financial \& competing interests disclosure}

The authors have no relevant affiliations or financial involvement with any organization or entity with a financial interest in or financial conflict with the subject matter or materials discussed in the manuscript. This includes employment, consultancies, honoraria, stock ownership or options, expert testimony, grants or patents received or pending, or royalties.

No writing assistance was utilized in the production of this manuscript.

\section{Bibliography}

1 Edelbroek P, van der Heijden J, Stolk L. Dried blood spot methods in therapeutic drug monitoring: methods, assays, and pitfalls. Ther. Drug Monitoring 31(3), 327-336 (2009).

2 Abbott R. European Bioanalysis Forum, 2nd open symposium: 'The broadening scope of validation: towards best practices in the world of bioanalysis'. Bioanalysis 2(4), 703-708 (2010).

3 Abbott R, Smeraglia J, White S et al. European Bioanalysis Forum workshop: Connecting strategies on dried blood spots. Sheraton Hotel, Brussels, Belgium 17-18 June 2010. Bioanalysis 2(11), 1809-1816 (2010).

4 Evans C. DIA/PhRMA workshop on dried blood spot sampling in the pharmaceutical industry. Bioanalysis 2(8), 1355-1359 (2010).

5 Spooner N, Lad R, Barfield M. Dried

blood spots as a sample collection technique for the determination of pharmacokinetics in clinical studies: considerations for the validation of a quantitative bioanalytical method. Anal Chem. 81, 1557-1563 (2009).

6 Rowland M, Emmons GT. Use of dried blood spots in drug development: pharmacokinetic considerations. AAPS J. 12(3), 290-293 (2010).

7 Knudsen R, Richmond JY, Slazyk W, Hannon H. Guidelines from the centers for disease control and prevention for the shipment of dried blood spot specimens. Infant Screening 16, 1-3 (1993).

- Website

101 Dangerous goods regulations.

www.iata.org/ps/publications/dgr/Pages/index.aspx 